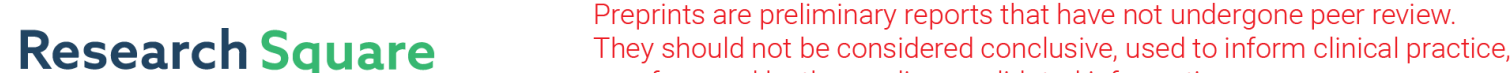 or referenced by the media as validated information.
}

\section{The deposition characteristics of Schistosoma japonicum eggs and its application in hatching method}

\section{yang man}

Shanghai Veterinary Research Institute Chinese Academy of Agricultural Sciences

\section{Rongyi Ji}

SHVRI: Shanghai Veterinary Research Institute Chinese Academy of Agricultural Sciences

\section{Yuanxi Shen}

SHVRI: Shanghai Veterinary Research Institute Chinese Academy of Agricultural Sciences

\section{Chuangang Zhu ( $\square$ zcg@shvri.ac.cn )}

Chinese Academy of Agricultural Sciences

\section{Short Report}

Keywords: Schistosoma japonicum, Density of eggs, Deposition characteristics, Hatching method

Posted Date: December 7th, 2020

DOI: https://doi.org/10.21203/rs.3.rs-121030/v1

License: (a) (i) This work is licensed under a Creative Commons Attribution 4.0 International License. Read Full License 


\section{Abstract \\ Background}

Schistosomiasis is an important zoonotic parasitic disease, which remains a major public concern in china. However, the detection of schistosomiasis in the field is still based on the traditional faecal hatching method, which is tedious and time-consuming. Therefore, method for detecting schistosomiasis in the field needs to be improved.

\section{Methods}

New Zealand rabbits artificially infected with $S$. japonicum cercariae were used as animal models to study the deposition characteristics of Schistosoma japonicum eggs. The distributions of eggs in the intestinal wall at $42 \mathrm{~d}$ and $60 \mathrm{~d}$ post-infection were compared. The distributions of eggs in rabbit faecal samples were also observed. Goat faeces were used to compare the conventional faecal hatching method and the simplified direct immersion faecal hatching method.

\section{Results}

The distribution of eggs in the intestinal wall in the animal model at $42 \mathrm{~d}$ post-infection was as follows: the number of eggs per gram (EPG) was the highest $(42780.13 \pm 4789.81 \mathrm{eggs} / \mathrm{g})$ in the rectum. The caecum had the largest proportion (42.97\%) of eggs deposited. At $60 \mathrm{~d}$ post-infection, the rectum still had the highest EPG $(117868.20 \pm 67232.80 \mathrm{eggs} / \mathrm{g})$. However, instead of the caecum, the lower colon had the largest proportion (64.90\%). Moreover, $42.20 \%$ of eggs occupied the periphery of rabbit faeces. In the comparison between the conventional faecal hatching method and the simplified direct immersion faecal hatching method, the direct faecal hatching method was simpler, and the results were similar to those of the conventional faecal hatching method.

\section{Conclusion}

The deposition characteristics of eggs and their distributions in faecal samples suggest that the direct faecal hatching method can be used to simplify routine faecal hatching detection.

\section{Background}

Schistosomiasis is an important zoonotic parasitic disease, and transmission has been reported in 78 countries[1-3]. More than 40 animals can be naturally infected with Schistosoma japonicum[4]. Livestock, the main reservoir host and source of infection of schistosomiasis japonicum, has been a key schistosomiasis control points in China[5]. 
In schistosomiasis-endemic areas, buffaloes and goats are the main livestock. Studies in the early period of schistosomiasis control in China indicated that buffaloes and goats were the most important reservoirs of disease transmission[6-9]. In recent years, goat breeding has expanded, though the rearing method still comprises traditional breeding of scattered populations. As goats are schistosomiasis infection sources, the prevalence rate and number of positive cases in goats have shown increasing trends. An improved detection method will facilitate rapid disease diagnosis and play an important role in schistosomiasis control[10,11]. However, current techniques for the detection of the disease have limitations.

The detection of schistosomiasis in the field is still based on the traditional faecal hatching method[1214]. Goat faeces have typical granular morphological characteristics. The conventional faecal hatching method requires faecal samples to be soft so that miracidia can emerge. Therefore, faecal samples are soaked until they are soft, and external force is subsequently applied to stir and mix the samples. After sieving, water is added to remove any faecal residue. This method is tedious and time-consuming. Therefore, method for detecting schistosomiasis in the field needs to be improved.

In this study, New Zealand rabbits, which produce faeces with morphological characteristics similar to those of goat faeces, were used as an animal model to investigate the distribution of $S$. japonicum eggs in the intestinal wall and faecal samples. A comparative experiment was conducted to elucidate the difference between the results of the direct faecal hatching method and the conventional hatching method. Revealing the distribution of schistosomiasis eggs in faeces will provide guidance for optimizing the hatching method.

\section{Materials And Methods}

\section{Host animals, parasites and samples}

Six healthy male New Zealand rabbits, each weighing approximately $4 \mathrm{~kg}$, were purchased from Shanghai Slake Experimental Animals. S. japonicum-infected snails were kept in the laboratory. Goat faeces were collected from 6 goat breeds in endemic areas that were positive for $S$. japonicum according to serology.

\section{Animal infections}

Six rabbits were fixed on a board, and their abdominal hair was shaved. The cercariae of S. japonicum were counted under an optical microscope. Each rabbit was infected with 1000 cercariae. Rabbits were divided into two groups: A and B. Group A was sacrificed and dissected after 42 days, and group B was sacrificed and dissected after 60 days.

\section{Sampling}

Faecal collection: Rabbit faeces were collected from the rectal segment and stored at $4^{\circ} \mathrm{C}$ for subsequent analysis. 
Intestinal wall samples: The whole intestine of rabbits was harvested and the external tissues, such as blood vessels and the mesangium, attached to the intestinal wall were separated and removed. According to intestinal function, the small intestine (including the duodenal jejunum and ileum) was divided into the caecum, colon and rectum. The small intestine was subdivided into upper, middle and lower segments. The colon was equally divided into upper and lower sections. The above samples were dissected; the intestinal contents were removed and sieved, and all the intestinal mucosal tissues were scraped with glass slides. The processed samples were placed in $50 \mathrm{ml}$ centrifuge tubes for weighing, and normal saline was added for homogenization.

\section{Intestinal tissue egg counts}

The above $800 \mu \mathrm{l}$ homogenate was digested with $10 \% \mathrm{NaOH}$ at $37^{\circ} \mathrm{C}$ for $2 \mathrm{~h}$, and $100 \mu \mathrm{l}$ was subsequently removed to count eggs under an optical microscope. Three biological replicates were performed for each sample, and 3 replicates were counted for each sample. The egg density and total number of eggs from each segment of the intestine were calculated.

\section{Frozen sectioning and observation of eggs in faecal samples}

The faecal pellets were frozen and sliced into sections with a thickness of $40 \mu \mathrm{m}$. Dye containing malachite green was added, and the sample was covered with a cover glass. After incubation for 0.5-1 hour at $37^{\circ} \mathrm{C}$, the distribution of eggs in the faecal samples was observed, recorded and photographed. Five hundred micrometres from the edge of the faecal pellet was defined as the periphery of the faecal pellet, while the remaining area was defined as the interior of the faecal pellet. The numbers of eggs in the faecal samples (periphery or interior faecal sphere) were counted, and the percentages were calculated.

\section{Egg hatching assay}

The collected faeces from schistosomiasis-positive goats were randomly assigned to four groups containing different goat faeces numbers $(10,20,40$ and 80$)$. Four samples of faeces were incubated directly or mixed after being crushed. Four replicates were conducted for each group for each method. The protocols are as follows.

Direct hatching method: A certain number of faecal pellets were placed directly into a $500 \mathrm{ml}$ hatching flask, and dechlorinated water was added to the height of the bottleneck. Loose absorbent cotton was

placed on the water surface in the bottle. Then, $10 \mathrm{ml}$ of distilled water was added slowly. The hatching flask was transferred into an incubator and incubated at $30^{\circ} \mathrm{C}$ for 6 hours. During this period, the liquid above the cotton was collected every 2 hours; this step was repeated three times. After sampling, dechlorinated water was supplemented to the previous volume and eggs were allowed to continue to hatch. 
Mixed hatching method: A certain number of faecal pellets were placed in a beaker, and dechlorinated water was added. The samples were fully mixed and subsequently mashed into a homogenate. After filtering the homogenate with a 60-mesh copper sieve, water was added until no obvious residual coarse fibre could be seen. The filter residue was resuspended in water and left for $20 \mathrm{~min}$ at room temperature for separation. The upper liquid phase was removed, and the residue was transferred into a $500 \mathrm{ml}$ hatching flask. The remaining steps were the same as those in the direct hatching method.

\section{Counts of S. japonicum miracidia}

The collected liquid was combined according to the different hatching methods. Formaldehyde was added and the solution was left overnight to fix the $S$. japonicum miracidia in the liquid. After the liquid was centrifuged at $4000 \mathrm{~g}$ for $10 \mathrm{~min}$, the supernatant was discarded, and the precipitate was collected. The miracidia were sequentially stained with 1-2 drops of iodine. The total number of miracidia was counted under an optical microscope.

\section{Statistical analysis}

SPSS 16.0 was used to analyse the experimental data. The arithmetic mean and standard deviation ( $\mathrm{x} \pm$ $s)$ of each indicator were calculated. Student's t test was employed to determine the statistical significance of the differences between the two hatching methods.

\section{Results}

\section{Distributions of deposited eggs in the intestinal wall during two post-infection periods}

Intestinal tract segmentation was performed in New Zealand white rabbits at $42 \mathrm{~d}$ and $60 \mathrm{~d}$ post-infection to count the egg density in the different segments of intestinal wall tissue. The numbers of eggs in the different segments of the intestinal wall varied greatly. The maximum egg density in rabbits at $42 \mathrm{~d}$ postinfection was observed in the rectum $(42780.13 \pm 4789.81)$, followed by the caecum $(20631.05 \pm$ $5554.82)$, lower segment of the small intestine (13545.3 \pm 12688.85$)$, lower colon (10864.51 \pm 1012.90$)$ and upper colon (9195.48 \pm 1783.13$)$. and upper segment of the small intestine $(2503.09 \pm 164.71)$. The lowest egg density was in the middle segment of the small intestine (519.34 \pm 74.48$)$. There were significant differences $(P<0.01)$ in the distribution densities of eggs in each segment of the intestinal wall. After 60 days of infection, the density of eggs in the intestinal wall of the rectum was still the highest (117868.2 \pm 67232.8$)$, followed by the lower colon (27813.92 \pm 25309.57$)$, the upper colon (15065.80 \pm 9471.56$)$, and the caecum $(13769.79 \pm 2867.65)$. The lowest density of eggs was deposited in the small intestine. The densities of eggs deposited in the upper small intestine (1621.76 \pm 57.69$)$, middle small intestine (1566.81 \pm 835.64), and lower small intestine (1615.42 \pm 1071.93$)$ showed little change and were distributed uniformly. The egg deposition densities in each segment are shown in Fig. 1. 
According to the number of eggs per gram of tissue (EPG) in each intestinal segment, the proportions of eggs per intestinal segment were calculated (Fig. 2).

After 42 days of infection, the numbers and proportions of eggs deposited in the intestinal walls of the infected animals were as follows: caecum $(792232,42.97 \%)$, lower small intestine $(350823,19.03 \%)$, rectum $(313364,16.99 \%)$, lower colon $(147702,11.66 \%)$, upper colon $(166362,5.59 \%)$, upper small intestine $(59354,3.21 \%)$ and middle small intestine $(14054,0.76 \%)$.

The numbers and proportions of eggs deposited in the intestinal walls of rabbits $60 \mathrm{~d}$ post-infection were as follows: rectum $(1116801,64.90 \%)$, caecum $(216357,12.57 \%)$, lower colon $(207213,12.04 \%)$, upper colon $(108473,6.30 \%)$, middle small intestine $(25715,1.49 \%)$ and lower small intestine $(25705,1.49 \%)$. The upper small intestine had the lowest number and proportion of deposited eggs (20657, $1.20 \%)$; the proportion of eggs deposited in the rectum significantly increased from $16.99-64.90 \%$.

\section{Investigation of the spatial distribution of eggs in faeces}

To obtain a visual impression of the distribution of the eggs in the faecal pellet, $60 \mathrm{~d}$ post-infection rabbit faeces were collected for frozen section observation. A total of 97 faecal pellets from different rabbits were collected, and six slices from each sample were observed. A total of $500 \mu \mathrm{m}$ from the edge of the pellet was designated as the periphery of the faecal pellet, and the remaining area was designated as the interior of the faecal pellet. The average number of eggs in the periphery of the faecal sphere was $0.12 \pm$ $0.46(57.10 \%)$, and the average number of eggs in the interior of the faecal pellet was $0.17 \pm 0.43$ (42.90\%). There was no significant difference between the number of eggs in the periphery and in the interior of the pellets $(P=0.15)$. The spatial distribution of the eggs is shown in Fig. 3 .

\section{Comparison of egg hatching methods in goat faecal samples collected from the field}

Faecal samples from goats infected with $S$. japonicum were collected on-site to compare the hatching rate between the direct hatching method and mixed hatching method. With the increase in the number of in faecal samples, the number of miracidia detected by the two methods showed an increasing trend, and there was no significant difference between the two methods $(P=0.127)$ (Table 1). 
Table 1

The two methods were compared for the hatching detection of goat faecal in the field

\begin{tabular}{|c|c|c|c|}
\hline \multirow{2}{*}{$\begin{array}{l}\text { The number of fecal } \\
\text { samples }\end{array}$} & \multicolumn{2}{|c|}{ The number of miracidia $(x \pm s)$} & \multirow[t]{2}{*}{$\mathrm{T}$ test value } \\
\hline & $\begin{array}{l}\text { Direct incubation } \\
\text { method }\end{array}$ & $\begin{array}{l}\text { Mixed incubation } \\
\text { method }\end{array}$ & \\
\hline 10 & $0.75 \pm 0.50$ & $0.50 \pm 0.58$ & 0.196 \\
\hline 20 & $1.75 \pm 0.96$ & $1.25 \pm 0.50$ & 0.196 \\
\hline 40 & $3.75 \pm 0.96$ & $2.25 \pm 1.26$ & 0.051 \\
\hline 80 & $5.0 \pm 0.82$ & $4.75 \pm 0.50$ & 0.319 \\
\hline
\end{tabular}

\section{Discussion}

S. japonicum mainly parasitizes the mesenteric vein and portal vein of the host. Reproductive success was investigated approximately 24 days after the host was infected with cercariae. Schistosoma eggs are mainly deposited in the liver and intestines, with only a small amount deposited in the mesenteric lymph nodes, pulmonary system, spleen and central nervous system $[15,16]$. The $S$. japonicum eggs deposited in the intestinal wall can cause inflammation in the tissues around the eggs, leading to ulceration and rupture of the intestinal wall. The Schistosoma eggs enter the intestinal cavity from the ruptured part of the intestinal wall and are excreted in the host's faeces. Therefore, eggs in the faeces can be detected to determine whether the host is infected with $S$. japonicum. The aetiology of livestock schistosomiasis is determined mainly by examining the miracidia hatched from the eggs in faecal samples. A certain number of faecal samples are collected and processed with the conventional incubation method; then, the samples are screened to remove factors that influence the results of the hatching method, such as related residues or pigments in the faecal samples. After hatching under specific conditions, the presence of infection and the degree of infection can be determined by observing the hatched miracidia.

Aetiological diagnosis of $S$. japonicum in domestic animals is important in the diagnosis of schistosomiasis, and the faecal hatching method is the basic and common method for aetiological diagnosis $[17,18]$. Some faecal samples from domestic animals, especially herbivorous livestock such as rabbits and goats, have special characteristics. The small intestine of domestic animals exhibits peristaltic movement, namely, segmented movement, which is mainly accomplished by circular muscle relaxation and contraction. After the absorption of water by the colon and rectum, a spherical faecal sample is formed. In the field of pathogen detection, there are differing opinions on the distribution of $S$. japonicum eggs in faeces[19-23]. Therefore, the challenge of how to analyse spherical faecal pellets has also become a controversial problem. The controversy revolves around whether it is necessary to crush the faecal pellet and incubate it. Some people think that crushing the pellet is required because eggs need to fully contact the hatching water to successfully hatch; however, others do not recommend crushing goat faeces, as there are many disadvantages after soaking. First, it increases the operation time. Second, it causes some eggs to be lost or hatch before bottling. Third, it increases the influence of faecal 
impurities in the hatching water, and fourth, it increases the turbidity of the hatching water, which affects the observation of miracidia.

The results showed that there was no significant difference in the hatching rate among 10 to 80 directincubated faecal pellets and the same amount of crushed faeces. However, the number of miracidia detected by the direct incubation method was higher than that detected by the conventional faecal hatching method.

An artificially infected $S$. japonicum animal model were employed to investigate the possible causes. First, we compared the distribution of deposited eggs of the Chinese mainland S. japonicum strain in the intestinal wall in rabbits at 42 and 60 days post-infection. The data showed that although the deposited eggs were distributed throughout the intestinal wall of the host, there were certain changes over time. The density of the eggs in the rectum was the highest, followed by the terminal tissues in the lower colon and caecum. Taking into account the size of each tissue segment, we calculated the proportion of eggs in each section of the intestinal wall and the egg distribution percentages according to the EPG results. The number of eggs deposited in the small intestine remained low during the course of the disease. The rectum and caecum accounted for more than $60 \%$ of the total eggs in the intestinal wall, especially the total number of eggs in the rectum, which accounted for $65 \%$ of the total eggs in the intestinal wall at 60 $\mathrm{d}$ post-infection. The faecal pellet is formed in the rectum, and the function of the rectum is mainly to absorb water. The eggs in the intestinal wall are rarely transferred to the faecal ball. It can be inferred that the eggs in the intestinal wall enter the faecal sample mainly in the large intestine, and the eggs in the rectum may enter the intestinal cavity when the intestinal wall is ruptured. They are mainly distributed in the periphery of the faecal sphere during the process of peristalsis and movement through the intestinal tract. During the necropsy of rabbits at $60 \mathrm{~d}$ post-infection, we also found that injuries to the rectal wall were more serious than those to the small intestine wall. Erosion, ulceration, irregular haemorrhage in the rectum, paving stone-like changes in the rectum and typical yellowish-grey nodules of $S$. japonicum eggs were observed. The faecal pellet can also cause mechanical friction, which leads to bleeding and allows eggs to enter the intestinal cavity.

According to the investigation of the distribution of schistosome eggs in the intestines of experimental rabbits, it was found that the egg density in the large intestine, especially the rectum, was the highest and the pathological damage was the most severe. The eggs in each segment of the intestine enter the faeces via the effect of intestinal peristalsis on chyme and digestive juice mixing. However, the spherical shape of the faeces is formed in the large intestine, especially the rectum; therefore, the eggs in the blood vessels or nodules are unlikely to be transferred to the inside of the faecal pellet; rather, they remain on the periphery of the faecal pellet. Considering the substantial total number of eggs in the rectal segment, it is concluded that it is feasible to hatch eggs in the faeces directly in water.

To observe the distributions of eggs in the faecal pellets, we used the section technique. The results showed that the distribution of eggs in a faecal pellet was consistent with that the distribution of eggs in the intestinal wall. There was no significant difference between the number of eggs the periphery and 
interior of the faecal pellet. Section observation revealed that although the surface of the spherical faecal pellet was smooth and uniform, the interior of the faecal pellet was loose and porous. It is speculated that during the incubation process, water easily permeates the interior of the faecal pellet so that eggs inside the faecal pellet can hatch successfully and the miracidia can emerge into the water.

It was demonstrated that the direct hatching method can be used for aetiological detection of schistosomiasis in spherical faecal samples. This method reduces the number of operational steps, such as mixing and crushing the faecal samples, and avoids the loss of eggs in the operational process. Moreover, the contamination of hatching water by pigments or toxins released after crushing the faecal samples can also be avoided with this method; this improves the observation of miracidia and thus has a prominent effect on detection. Therefore, this method is suitable for the qualitative detection of goat schistosomiasis on-site.

\section{Conclusions}

The distribution of schistosomiasis eggs in faeces was elucidated, and the hatching method was optimized. The detection results of the direct faecal hatching method were basically consistent with those of the mixed hatching method $(P=0.127)$. The simplicity of this method makes it suitable for on-site goat schistosomiasis detection.

\section{Abbreviations}

EPG: eggs per gram; S. japonicum:Schistosoma japonicum.

\section{Declarations}

\section{Ethics approval}

This study was carried out in strict accordance with the recommendations in the Animal Ethics Procedures and Guidelines of the People's Republic of China. The protocol was approved by the Committee on the Ethics of Animal Experiments of Shanghai Veterinary Research Institute, Chinese Academy of Agricultural Sciences (Protocol Number: SHVRI-SZ-20200724-01).

\section{Consent for publication}

Not applicable

\section{Availability of data and material}

The datasets used or analysed in the current study are available from the corresponding author upon reasonable request.

\section{Competing interests}


The authors declare that they have no competing interests.

\section{Funding}

This study was financially supported by the Schistosoma Vaccine Subprogram of the Key Research and Development Program to Jiaojiao Lin and Zhiqiang Fu (Grant No. 0301001999107), Fundamental Research Funds to Chuangang Zhu (Grant No. 0201007002008) and the Hong Yang Schistosoma Application Research Team Innovation Project (Grant No. 0201007001011).

\section{Authors' contributions}

$C Z, M Y, Y S$, and RJ designed the study. CZ, MY, YS and RJ provided technical input for the experiments. MY, RJ and YS wrote the manuscript. MY performed the statistical analysis. All the authors read and approved the final manuscript.

\section{Acknowledgements}

The authors wish to thank the Shanghai Veterinary Research Institute for facilitating the implementation of this study.

\section{Authors' information}

Key Laboratory of Animal Parasitology, Ministry of Agriculture of China, Shanghai Veterinary Research Institute, Chinese Academy of Agricultural Sciences, Shanghai, China.

\section{References}

1. Steinmann P, Keiser J, Bos R, Tanner M, Utzinger J. Schistosomiasis and water resources development: systematic review, meta-analysis, and estimates of people at risk. The Lancet Infectious Diseases. 2006;6(7):411-25.

2. Colley DG, Bustinduy AL, Secor WE, King $\mathrm{CH}$. Human schistosomiasis. The Lancet. 2014;383(9936):2253-64.

3. Hotez PJ, Alvarado M, Basanez MG, Bolliger I, Bourne R, Boussinesq M, et al. The global burden of disease study 2010: interpretation and implications for the neglected tropical diseases. PLoS Negl Trop Dis. 2014;8(7):e2865.

4. Wang TP, Shrivastava J, Johansen MV, Zhang SQ, Wang FF, Webster JP. Does multiple hosts mean multiple parasites? Population genetic structure of Schistosoma japonicum between definitive host species. Int J Parasitol. 2006;36(12):1317-25.

5. Long-De Wang MD, Hong-Gen Chen, Ph.D., Jia-Gang Guo, Ph.D., Xiao-Jun Zeng, M.D., Xian-Lin Hong, Ji-Jie Xiong, Xiao-Hua Wu, M.Sc., Xian-Hong Wang, Ph.D., Li-Ying Wang, Gang Xia, M.Sc., Yang Hao, M.Sc., Daniel P. Chin, M.D., and Xiao-Nong Zhou, Ph.D. A Strategy to Control Transmission of Schistosoma japonicum in china. 2009;360(2):121-8 
6. Li YS, McManus DP, Lin DD, Williams GM, Harn DA, Ross AG, et al. The Schistosoma japonicum selfcure phenomenon in water buffaloes: potential impact on the control and elimination of schistosomiasis in China. Int J Parasitol. 2014;44(3-4):167-71.

7. Gray DJ, Williams GM, Li Y, Chen H, Forsyth SJ, Li RS, et al. A cluster-randomised intervention trial against Schistosoma japonicum in the Peoples' Republic of China: bovine and human transmission. PLoS One. 2009;4(6):e5900.

8. Cao Z, Huang Y, Wang T. Schistosomiasis Japonica Control in Domestic Animals: Progress and Experiences in China. Front Microbiol. 2017;8:2464.

9. S.T. McGarvey XNZ, A.L. Willingham III, Z. Feng and R. Olveda. The Epidemiology and Host-Parasite Relationships of Schistosoma japonicum in Definitive Hosts. 1999.

10. Lv C, Hong Y, Fu Z, Lu K, Cao X, Wang T, et al. Evaluation of recombinant multi-epitope proteins for diagnosis of goat schistosomiasis by enzyme-linked immunosorbent assay. Parasit Vectors. 2016;9:135.

11. Guo Q, Chen C, Zhou K, Li Y, Tong L, Yue Y, et al. Evaluation of a real-time PCR assay for diagnosis of schistosomiasis japonica in the domestic goat. Parasit Vectors. 2020;13(1):535.

12. Wang WQ YQ, Peng YL. Clinical application of three schistosomiasis detection methods Animal and Poultry Industry. 2010;(04):66-7.

13. Alarcon de Noya B, Ruiz R, Losada S, Colmenares C, Contreras R, Cesari IM, et al. Detection of schistosomiasis cases in low-transmission areas based on coprologic and serologic criteria The Venezuelan experience. Acta Trop. 2007;103(1):41-9.

14. Yu JM, de Vlas SJ, Jiang QW, Gryseels B. Comparison of the Kato-Katz technique, hatching test and indirect hemagglutination assay (IHA) for the diagnosis of Schistosoma japonicum infection in China. Parasitol Int. 2007;56(1):45-9.

15. Ito J. Studies on the host-parasite relationships of Schistosoma japonicum in common laboratory animals. Jpn J Med Sci Biol. 1955;8(1):43-62.

16. Vogel H, Minning W. [Acquired resistance of Macacus rhesus to Schistosoma japonicum]. Z Tropenmed Parasitol. 1953;4(4):418-505.

17. Wang XF CA, Zhou ML. Current status of experimental diagnosis of schistosomiasis. International journal of laboratory medicine. 2016;37(13):1824-6.

18. Nelwan ML. Schistosomiasis: Life Cycle, Diagnosis, and Control. Curr Ther Res Clin Exp. 2019;91:5-9.

19. Yu JM, Yuan HC, Chen QM. Negative binomial distribution of eggs of schistosoma japonicum in stool. Chin j preventive medicine. 1996;30(6):360-3

20. Yu JM, Chen QM, Yuan HC. Distribution of schistosoma japonicum egg in the stools. Chinese journal of parasitic diseases control. 1998;11(04):3-5.

21. Ratard RC, Kouemeni L, Ekani Bessala MM, Ndamkou NC. Distribution and preservation of Schistosoma mansoni eggs in stools. J Trop Med Hyg. 1990;93(6):413-6. 
22. Shen Xue-Hui DJ-R, Sun Le-Ping , Fu Zhong-Yu , Li Ye-Fang, Qu Guo-Li , Wang Wei , Xing Yun-Tian ,

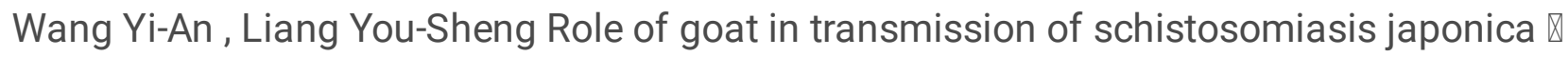
Schistosome development in goat and egg count and distribution in goat feces. Chinese journal of schistosomiasis prevention and control. 2016;28(05):502-6.

23. Hirose Y, Matsumoto J, Kirinoki M, Shimada M, Chigusa Y, Nakamura S, et al. Schistosoma mekongi and Schistosoma japonicum: Differences in the distribution of eggs in the viscera of mice. Parasitol Int. 2007;56(3):239-41.

\section{Figures}



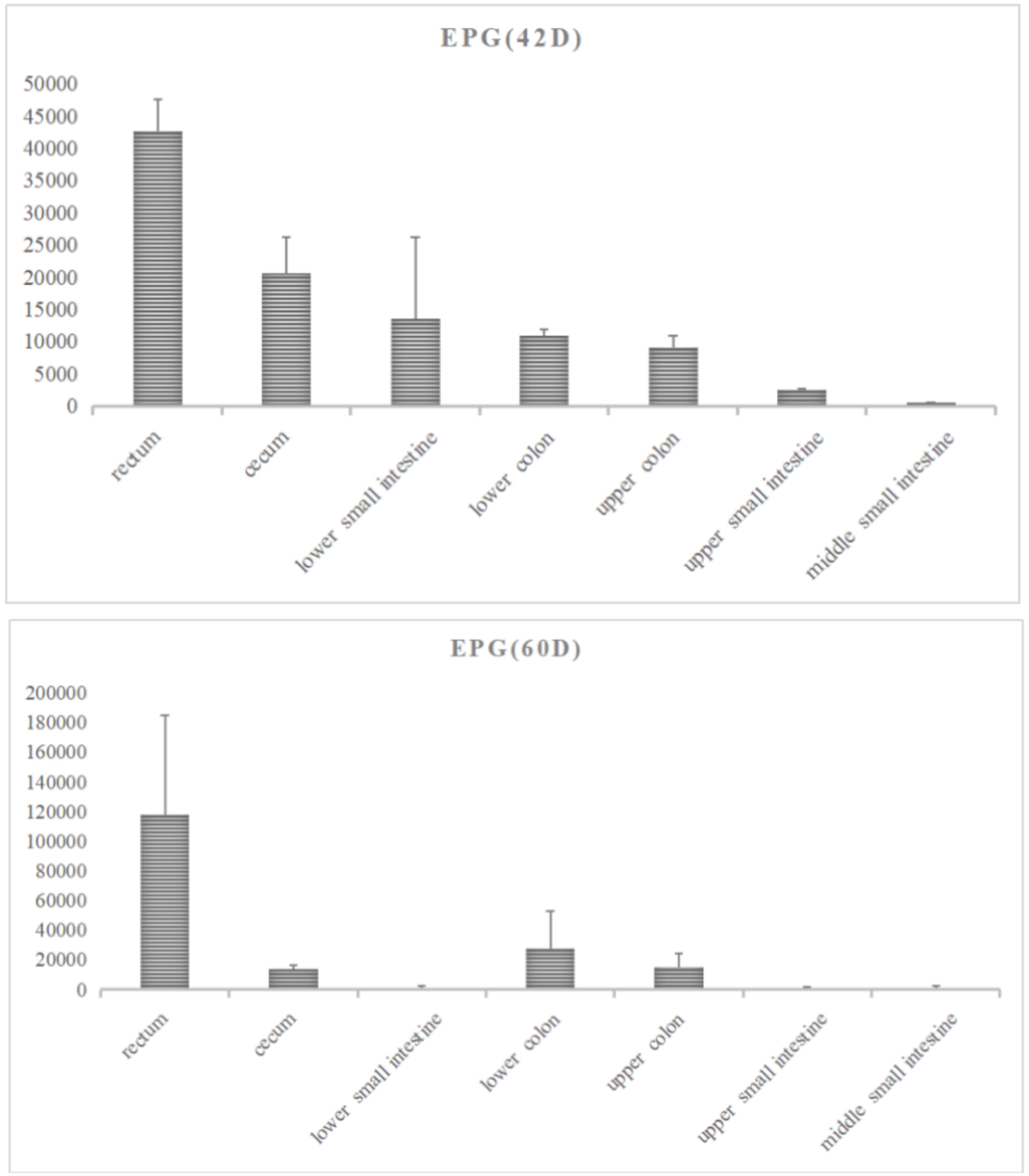

\section{Figure 1}

The density of eggs deposited in different parts of the intestinal wall. A: Infection of $42 \mathrm{~d}$, B: Infection of $60 \mathrm{~d}$. 

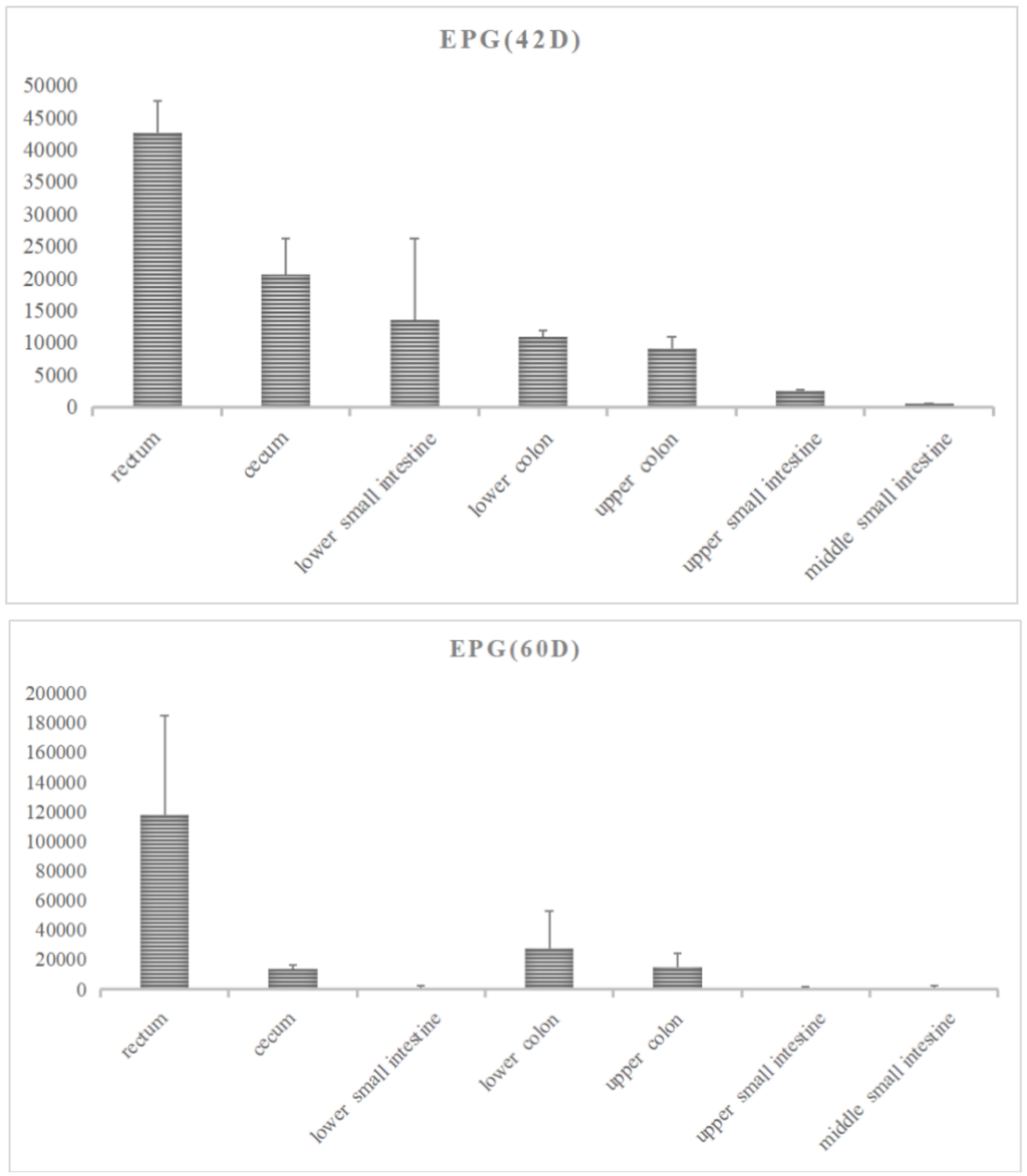

\section{Figure 1}

The density of eggs deposited in different parts of the intestinal wall. A: Infection of $42 \mathrm{~d}$, B: Infection of $60 \mathrm{~d}$. 


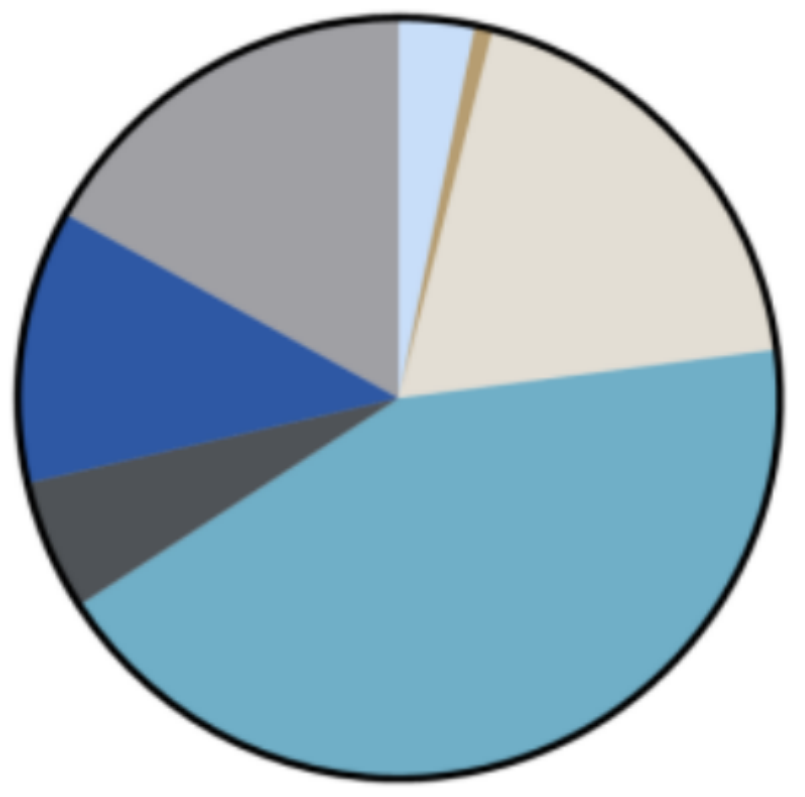

$\square 3.21 \%$ upper small intestine

$\square 0.76 \%$ middle small intestine

$\square 18.99 \%$ lower small intestine

口 $42.88 \%$ caecum

$\square 5.58 \%$ upper colon

$\square 11.63 \%$ lower colon

$\square 16.95 \%$ rectum

A

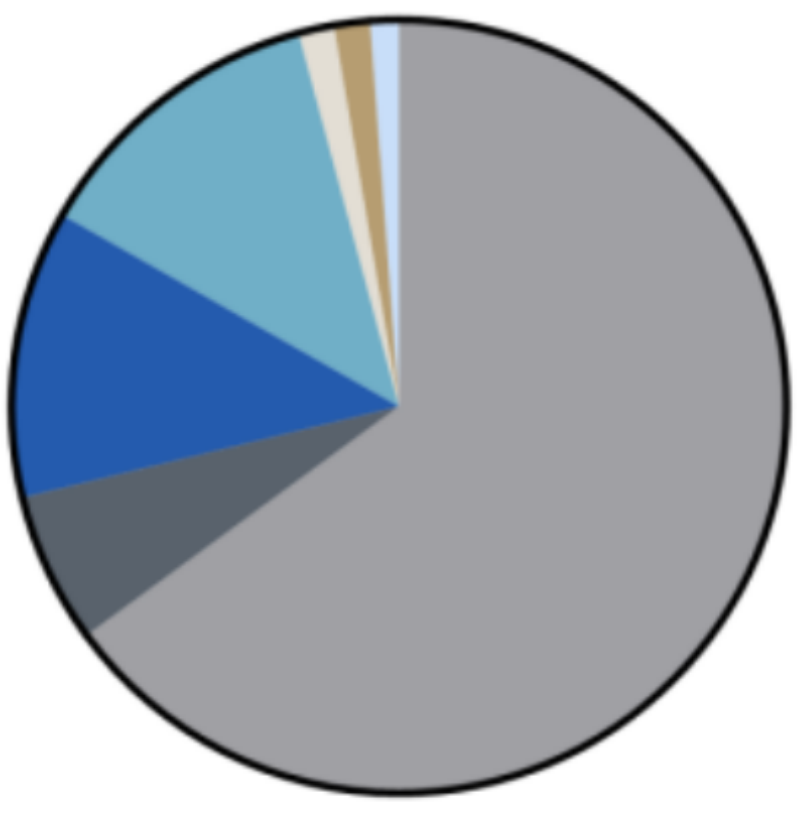

$\square 1.20 \%$ upper small intestine

口 $1.49 \%$ middle small intestine

ㅁ $1.49 \%$ lower small intestine

ㅁ $12.57 \%$ caecum

$\square 12.04 \%$ lower colon

$\square 6.30 \%$ upper colon

口 $64.91 \%$ rectum

B

Figure 2

The percentage of eggs deposited in different parts of intestinal wall tissue. A: Infection of $42 \mathrm{~d}$, B: Infection of $60 \mathrm{~d}$. 


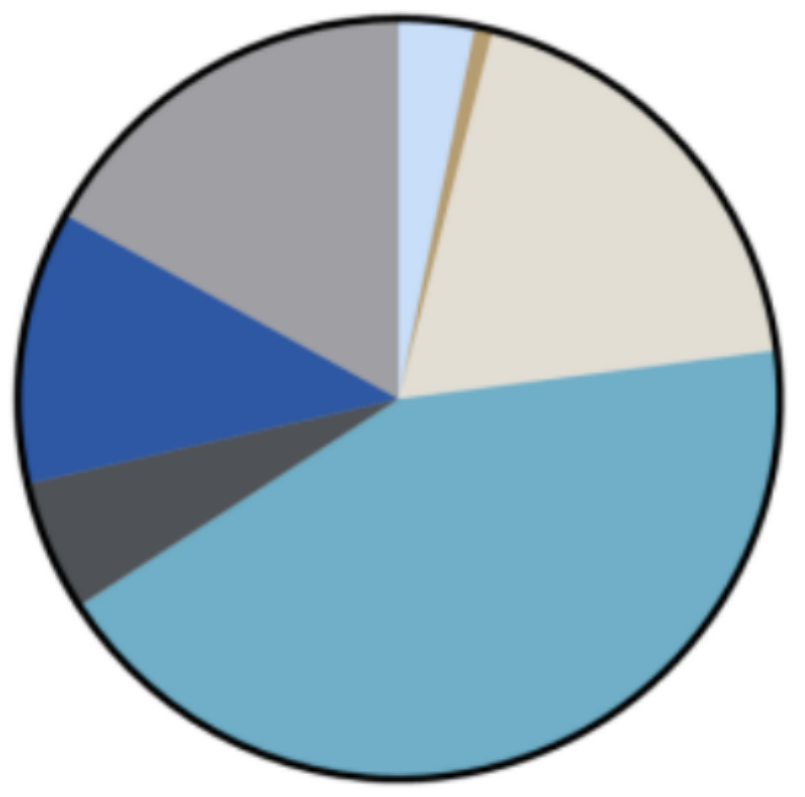

$\square 3.21 \%$ upper small intestine

口 $0.76 \%$ middle small intestine

$\square \quad 18.99 \%$ lower small intestine

口 $42.88 \%$ caecum

$\square 5.58 \%$ upper colon

$\square 11.63 \%$ lower colon

$\square 16.95 \%$ rectum

$\square 1.20 \%$ upper small intestine $\square 1.49 \%$ middle small intestine 口 $1.49 \%$ lower small intestine ㅁ $12.57 \%$ caecum

口 $12.04 \%$ lower colon

$\square 6.30 \%$ upper colon

口 $64.91 \%$ rectum

B

Figure 2

The percentage of eggs deposited in different parts of intestinal wall tissue. A: Infection of $42 \mathrm{~d}$, B: Infection of $60 \mathrm{~d}$. 

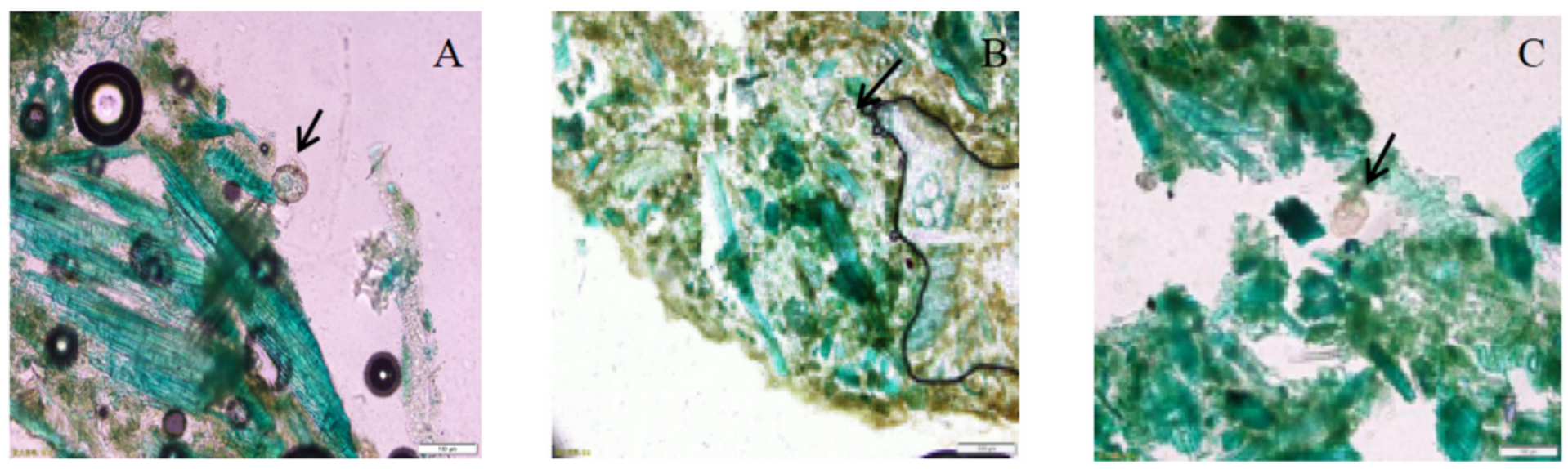

Figure 3

Distribution of eggs in faecal pellet A: Eggs on the edge of the faecal pellet (10x); B: Eggs between the edge and interior of the faecal pellet (5x); C: Eggs on the interior of the faecal pellet (10x)
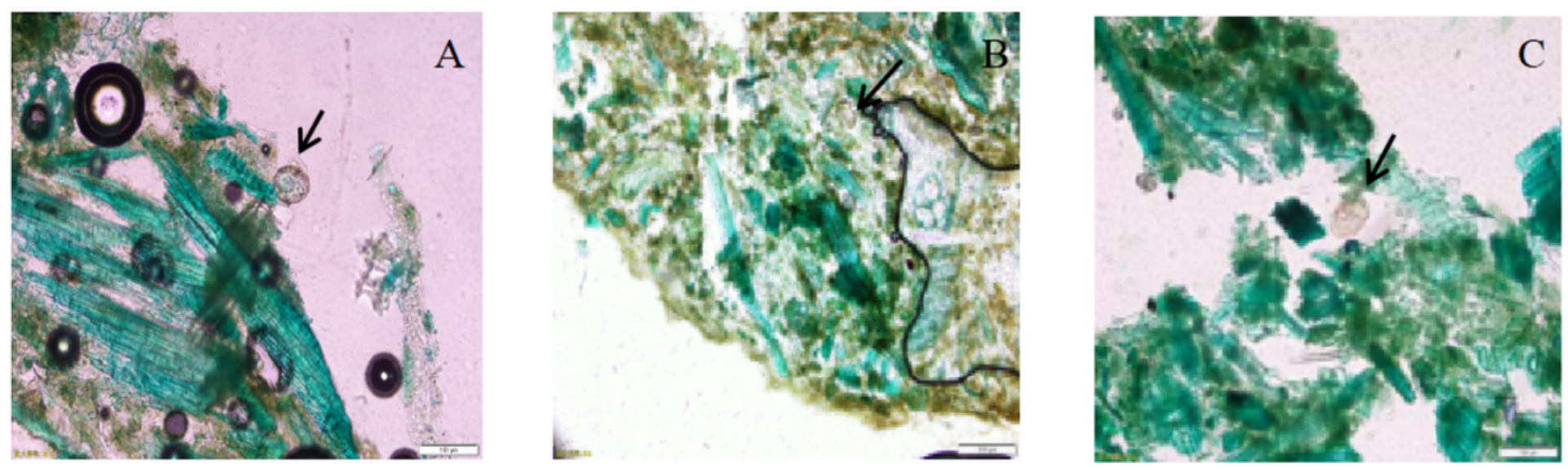

\section{Figure 3}

Distribution of eggs in faecal pellet A: Eggs on the edge of the faecal pellet (10x); B: Eggs between the edge and interior of the faecal pellet (5x); C: Eggs on the interior of the faecal pellet (10x) 